\title{
Influence of High-k Gate Dielectric on Nanoscale DG-MOSFET
}

\author{
S. K. Mohapatra ${ }^{1 *}$, K. P. Pradhan ${ }^{1}$ and P. K. Sahu ${ }^{1}$ \\ ${ }^{1}$ Dept. of Electrical Engineering, National Institute of Technology, Rourkela, - \\ 769008, Odisha, India
}

Skmctc74@gmail.com,kp2.etc@gmail.com,pksahu@nitrkl.ac.in

\begin{abstract}
Influence of dielectric materials as gate oxide on various short channel device parameters using a 2-D device simulator has been studied in this paper. It is found that the use of high-k dielectrics directly on the silicon wafer would degrade the performance. This degradation is mainly due to the fringing field effect developed from gate to source/drain. This fringing field will further generate electric field into the channel region from sourceldrain region which weakens the gate control. Therefore, by taking the gate stack engineering into account it has been shown that the induced electric field along the channel can be minimized as well as the device performance can be enhanced. This paper examined various parameters of the device like potential distribution from source and drain, threshold voltage $\left(V_{t h}\right)$, drain induced barrier lowering (DIBL), subthreshold slope $(S S)$, on-current $\left(I_{\text {on }}\right)$, off-current $\left(I_{\text {off }}\right)$ and Transconductance $\left(g_{m}\right)$ by taking different dielectric materials [SiO ${ }_{2}(\varepsilon=3.9), \mathrm{Si}_{3} \mathrm{~N}_{4}$ ( $\varepsilon=7.5)$, $\mathrm{HfO}_{2}(\varepsilon=24)$ and $\left.\mathrm{Ta}_{2} \mathrm{O}_{5}(\varepsilon=50)\right]$ as gate oxide $(s)$.
\end{abstract}

Keywords: Double gate MOSFET, High-k dielectric, Gate stack, Short channel effects (SCES), Equivalent oxide thickness (EOT)

\section{Introduction}

The major limitation in scaling of CMOS devices is the gate dielectric thickness [1 3]. The conventional $\mathrm{SiO}_{2}$ as gate dielectric has reached the point where film thickness is only a few atomic layers thick. At these dimensions or below $1.5 \mathrm{~nm}$, the direct tunnelling dramatically increases the leakage current which, consequently increases the static power and hence affect the circuit operation as suggested by CAMPBELL et al. \& RIBES et al. [4-5]. To get rid of this critical problem, high-k dielectrics have been introduced by taking directly on the silicon wafer or on the $\mathrm{SiO}_{2}$ layer. While keeping the equivalent oxide thickness (EOT) constant, high-k dielectrics permits the increase in physical thickness (a factor of $\mathrm{k} / \mathrm{k}_{\mathrm{SiO} 2}$ ) of the gate oxide to inhibit gate tunnelling [5-6]. However, it has been verified by device simulation that the use of high-k gate dielectrics directly on silicon wafer would degrade the short channel performance. This degradation is mainly caused by the fringing fields either from the gate to the source/drain regions or from the source/drain to the channel region which weakens the gate control [7]. But these shortcomings to some extent can be overcome by taking the gate stack (GS) configuration, i.e., high-k dielectrics over $\mathrm{SiO}_{2}$ layer [8-11]. By taking a thick layer of dielectric material over a thin $\mathrm{SiO}_{2}$ layer keeping EOT constant significantly reduces the gate tunnelling current.

In addition, there are various limitations with the use of high-k gate dielectrics : 1) the use of high-k dielectrics results in poly silicon gate depletion effects and finite inversion layer 
(FIL) capacitance [6]. Gate depletion in current poly silicon gate technology increases the effective gate thickness and degrades the device performance. Therefore, to mitigate this problem, it is desirable to replace the poly silicon gate with mid-gap gates or metal gates. 2) Another challenge for integrating high-k is the threshold voltage stability during operation. The shift in $\mathrm{V}_{\text {th }}$ known as hysteresis phenomenon is attributed to trapping of charges in the pre-existing traps without creation of additional traps. This problem is somewhat mitigated by considering the Hafnium (Hf)-based dielectrics [4].In this work, extensive simulations have been carried out to study the performance degradation due to fringing fields in high-k dielectrics and its minimization by considering gate stack engineering. Different GS configurations are simulated to optimize the device performance by integrating high-k dielectrics into various structures..

\section{Device Design and Structure}

The schematic structure of DG MOSFET is shown in Fig. 1. In this structure both the channel length $(\mathrm{L})$ and Source/Drain length $\left(\mathrm{L}_{\mathrm{S}} / \mathrm{L}_{\mathrm{D}}\right)$ are fixed as $40 \mathrm{~nm}$, the silicon thickness $\left(\mathrm{t}_{\mathrm{Si}}\right)$ is taken as $10 \mathrm{~nm}$ and a uniform density of $\mathrm{N}_{\mathrm{D}}$ is taken as $10^{20} \mathrm{~cm}^{-3}$. The channel is doped $\left(\mathrm{N}_{\mathrm{A}}\right)$ with $10^{16} \mathrm{~cm}^{-3}$. Two different types of structural models have been considered. The first model is structured considering single oxide layer and the second with double oxide layer or gate stack. In each case the EOT is fixed at $1.1 \mathrm{~nm}$. To maintain a constant capacitance for $\mathrm{k}=7.5, \mathrm{k}=24$ and $\mathrm{k}=50$, the corresponding high $-\mathrm{k}$ thicknesses are $2.1 \mathrm{~nm}, 6.8 \mathrm{~nm}$ and $14.1 \mathrm{~nm}$ respectively for single layer configurations. Further for double layer/GS configurations $\mathrm{SiO}_{2}$ layer thickness is fixed at $0.6 \mathrm{~nm}$ and above this layer $0.5 \mathrm{~nm}$ equivalent thickness of high-k layer is deposited, so that the EOT reaches $1.1 \mathrm{~nm}$. This work considers the high-k dielectrics as $\mathrm{Si}_{3} \mathrm{~N}_{4}, \mathrm{HfO}_{2}$ and $\mathrm{Ta}_{2} \mathrm{O}_{5}$ and the work function for the gate material is taken as $4.8 \mathrm{eV}$.

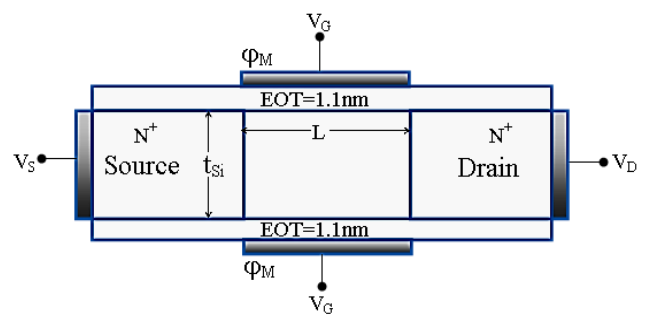

Figure 1. Schematic structure of Double Gate N-MOSFET

\section{Device Simulation}

To obtain accurate results for MOSFET simulation we need to account for the mobility degradation that occurs inside inversion layers. The degradation is normally occurs as a result of higher surface scattering near the semiconductor to insulator interface. So in the simulation the inversion-layer Lombardi constant voltage and temperature (CVT) mobility model was considered. The Shockley-Read-Hall (SRH) model simulates the leakage currents that exist due to thermal generation. Electrons in thermal equilibrium at given temperature with a semiconductor lattice obey Fermi-Dirac statistics. The use of Boltzmann statistics is normally justified in semiconductor device theory, however, Fermi-Dirac statistics are necessary to account for certain properties of very highly doped (degenerate) materials. The model FermiDirac uses a Rational Chebyshev approximation that gives results close to the exact values. Auger recombination models for minority carrier recombination have been used. 
Furthermore, we chose Gummel's method (or the decoupled method) which performs a Gummel iteration for Newton solution [12].

\section{Results and Discussion}

In Figure $2 \& 3, \mathrm{I}_{\mathrm{D}}-\mathrm{V}_{\mathrm{GS}}$ transfer characteristics have been shown on linear scale and $\log$ scale for different device structures. Here different dielectric materials are considered as gate oxide for both single and double layer configurations and their characteristics have been analyzed for $\mathrm{V}_{\mathrm{DS}}=0.1 \mathrm{~V}$ and $1.2 \mathrm{~V}$. The Sub threshold Slope (SS) is the major parameter for calculating the off state current. Furthermore, SS is calculated as:

$$
S S(m V / \text { dec })=\frac{\partial V_{G}}{\partial\left(\log I_{D}\right)}
$$

The typical value for the SS of Multigate MOSFET is $60 \mathrm{mV} /$ decade. In this paper the $\mathrm{SS}$ is extracted by calculating the inverse of maximum slope of $\mathrm{V}_{\mathrm{GS}}$ versus $\log \left(\mathrm{I}_{\mathrm{D}}\right)$ curve as shown in the TABLE 1 . Therefore, comparing the values in TABLE 1 , it is clear that with increasing high-k dielectric permittivity for the single oxide layer configurations, the SS value increases and reaches a value $75.53 \mathrm{mV} /$ decade. But, for double layer/gate stack configuration the SS value nearly approaches the ideal value.
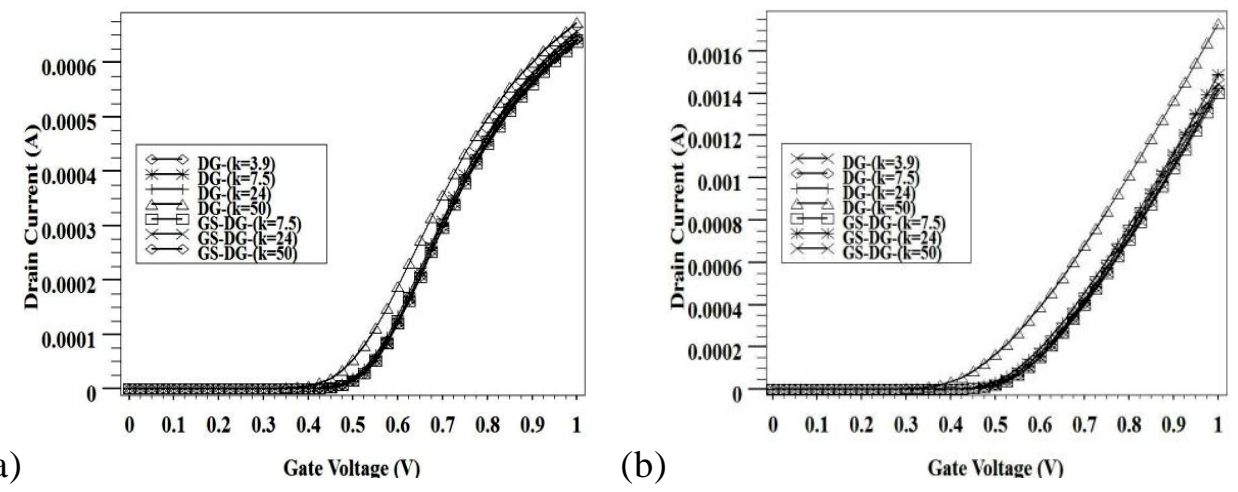

Figure 2. Behaviour of Drain Current $\left(I_{D}\right)$ as a function of Gate Voltage ( $\left.V_{G S}\right)$ in linear scale at (a) $V_{D S}=0.1 \mathrm{~V}$ (b) $V_{D S}=1.0 \mathrm{~V}$

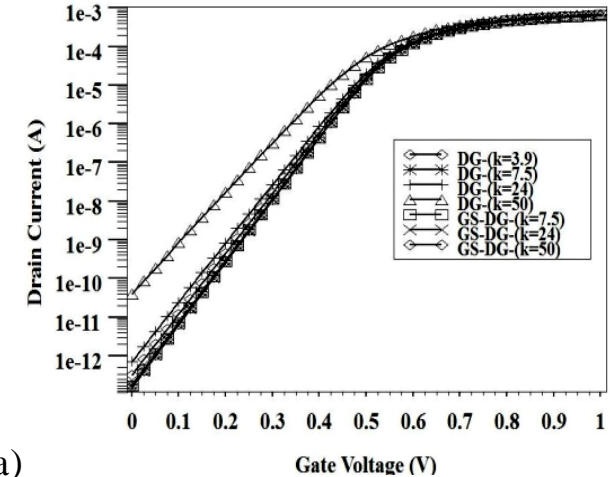

(a)

Figure

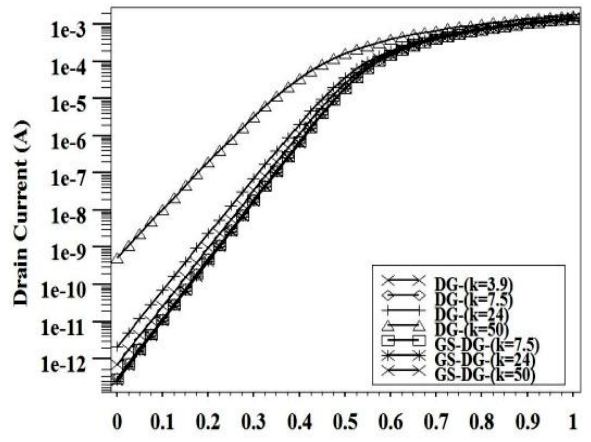

(b)
Gate Voltage (V)

in of Drain Current $\left(I_{D}\right)$ as a function of $G a t$
scale at (a) $V_{D S}=0.1 V$ (b) $V_{D S}=1.0 \mathrm{~V}$ 
The $\mathrm{V}_{\text {th }}$ is also a very important parameter for higher on state current. The $\mathrm{V}_{\text {th }}$ is amount of $V_{G S}$ when the $I_{D}$ is equal to $1 \times 10^{-6} \mathrm{~A}$. The extraction is given in Table 1 . It is observed that for single oxide layer configuration at $\mathrm{k}=50$ shows higher $\mathrm{I}_{\mathrm{on}}$. But, at the same time it also offers a undesirable higher leakage current $\left(\mathrm{I}_{\text {off }}\right)$ in the order of 59pA. The $\mathrm{g}_{\mathrm{m}}$ versus $\mathrm{V}_{\mathrm{GS}}$ characteristics have been compared for different device structures as shown in Fig.4. As we know:

$$
g_{m}=\partial I_{D} / \partial V_{G S}
$$

The value of $g_{m}$ is extracted by taking the derivative of $I_{D}-V_{G S}$ curve \& the values are summarized in Table 2. It is observed from the said table that the GS configurations are showing higher values of $\mathrm{g}_{\mathrm{m}}$.
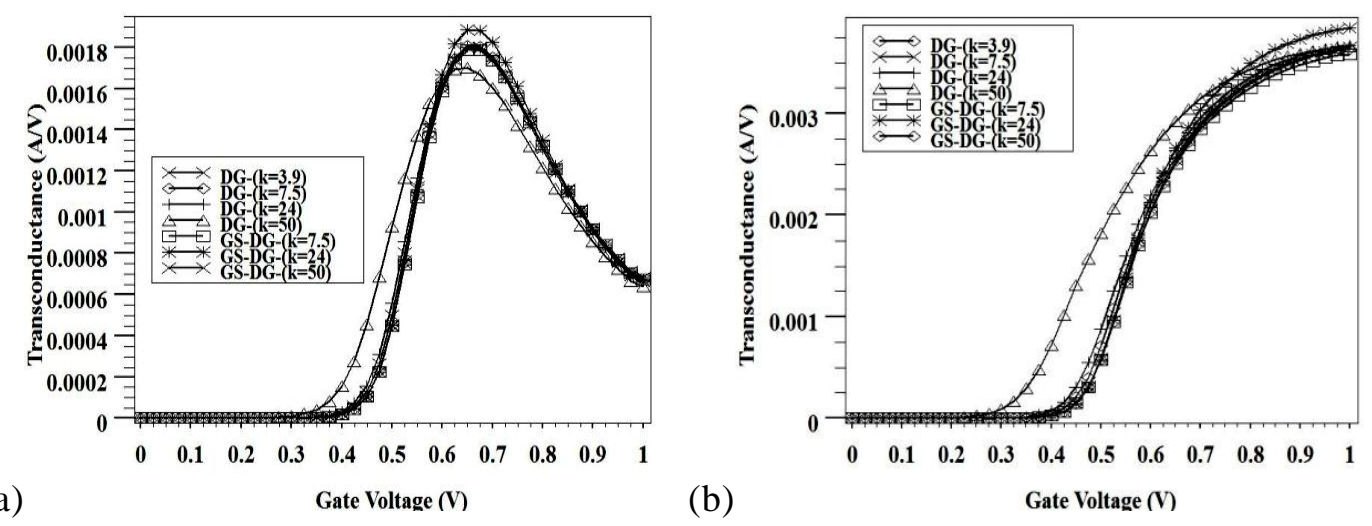

Figure 4. Variation of transconductance $\left(g_{m}\right)$ with $V_{G s}$ for different configurations at (a) $V_{D S}=0.1 \mathrm{~V}$ (b) $V_{D S}=1.0 \mathrm{~V}$

The value of DIBL is calculated as per the relation :

$$
D I B L=\frac{\Delta V_{t h}}{\Delta V_{D S}}
$$

The GS structures shows better values of DIBL as compared to single layer structures.

\begin{tabular}{|c|c|c|c|c|c|c|}
\hline Configuration & $\mathrm{SS}(\mathrm{mV} / \mathrm{dec})$ & $\mathrm{I}_{\mathrm{on}}(\mathrm{mA} / \mu \mathrm{m})$ & $\mathrm{V}_{\text {th }}$ & $\mathrm{SS}(\mathrm{mV} / \mathrm{dec})$ & $\mathrm{I}_{\mathrm{on}}(\mathrm{mA} / \mathrm{um})$ & $\mathrm{V}_{\text {th }}$ \\
\hline & \multicolumn{3}{|c|}{ At $V_{D S}=0.1 \mathrm{~V}$} & \multicolumn{3}{|c|}{ At $V_{D S}=1 \mathrm{~V}$} \\
\hline DG k=3.9 & 62.01 & 0.64 & 0.422 & 62.22 & 1.41 & 0.409 \\
\hline DG k=7.5 & 62.15 & 0.64 & 0.421 & 62.32 & 1.42 & 0.407 \\
\hline DG k=24 & 65.13 & 0.65 & 0.403 & 65.37 & 1.48 & 0.377 \\
\hline DG k=50 & 75.53 & 0.67 & 0.337 & 76.34 & 1.73 & 0.255 \\
\hline GS-DG k=7.5 & 62.06 & 0.64 & 0.422 & 62.25 & 1.40 & 0.408 \\
\hline GS-DG k=24 & 61.80 & 0.66 & 0.423 & 61.94 & 1.49 & 0.410 \\
\hline GS-DG k=50 & 63.42 & 0.64 & 0.412 & 63.67 & 1.44 & 0.395 \\
\hline
\end{tabular}

Table 1. Extracted Device Parameters for Different Cofigurations 
The $I_{\text {off }}$ is extracted by calculating the $I_{D}$ at $V_{G S}=0$ and $V_{D S}=V_{D D}$. It is important to keep $I_{\text {off }}$ very small in order to minimize the device static power dissipation. From TABLE 2, it is clear that the $\mathrm{I}_{\text {off }}$ value is very low for GS configuration which is one of the desired performance of the Device.

Table 2. Extracted Parameters

\begin{tabular}{ccccc}
\hline Configuration & $\mathrm{I}_{\text {off }}(\mathrm{pA})$ at $\mathrm{V}_{\mathrm{DS}}=0.2 \mathrm{~V}$ & $\begin{array}{c}\mathrm{g}_{\mathrm{m}}(\mathrm{mS}) \\
\text { at } \mathrm{V}_{\mathrm{DS}}=0.1 \mathrm{~V}\end{array}$ & $\begin{array}{c}\mathrm{g}_{\mathrm{m}}(\mathrm{mS}) \\
\text { at } \mathrm{V}_{\mathrm{DS}}=1 \mathrm{~V}\end{array}$ & DIBL $(\mathrm{mV} / \mathrm{V})$ \\
\hline \hline DG $(\mathrm{k}=3.9)$ & 0.393 & 1.81 & 3.63 & 14.863 \\
\hline DG $(\mathrm{k}=7.5)$ & 0.397 & 1.82 & 3.64 & 15.019 \\
\hline DG $(\mathrm{k}=24)$ & 0.845 & 1.81 & 3.66 & 29.342 \\
\hline DG $(\mathrm{k}=50)$ & 59.028 & 1.71 & 3.65 & 90.308 \\
\hline GS-DG k=7.5) & 0.394 & 1.80 & 3.58 & 14.937 \\
\hline GS-DG $(\mathrm{k}=24)$ & 0.390 & 1.90 & 3.84 & 14.254 \\
\hline GS-DG $(\mathrm{k}=50)$ & 0.430 & 1.81 & 3.63 & 19.269 \\
\hline
\end{tabular}

Figure 5 represents the variations of DIBL, $\mathrm{V}_{\text {th }}$ and $\mathrm{SS}$ values as a function of dielectric permittivity (k) for both single layer and double layer configurations

(a)
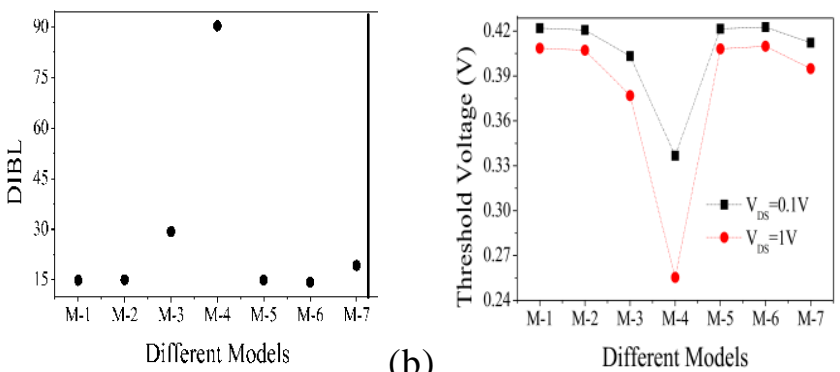

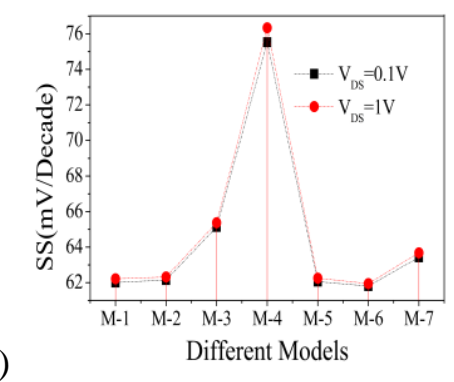

(c)
Figure 5. Variation of DIBL (a), Threshold Voltage (b) and SS (c) as a function of gate dielectric permittivity $(k)$ for different configurations

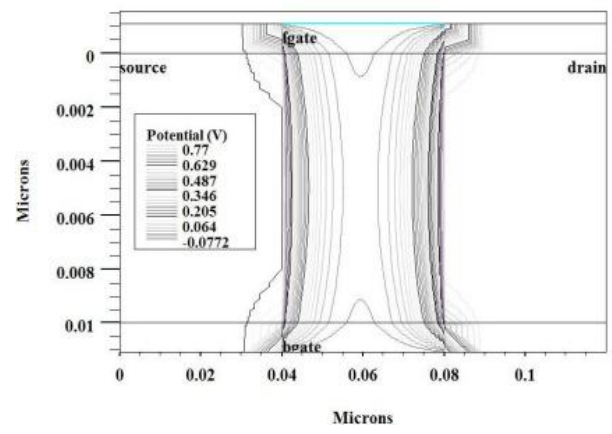

(a) $\mathrm{k}=3.9$

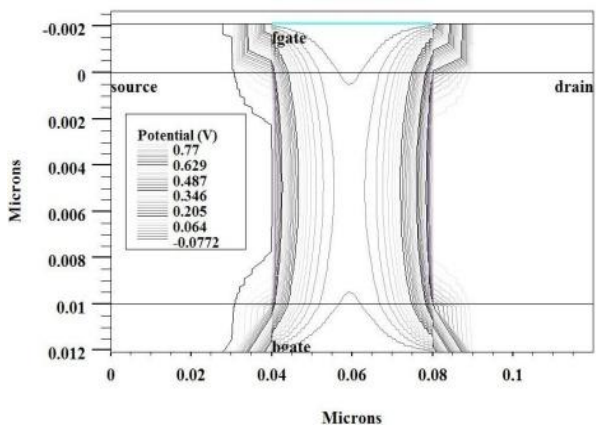

(b) $\mathrm{k}=7.5$ 


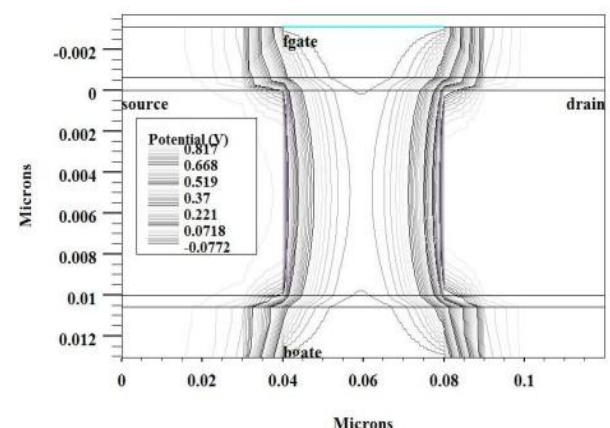

(c) $\mathrm{k}=24$

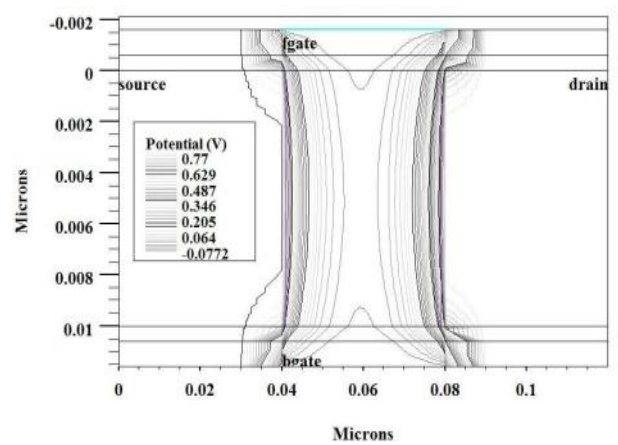

(e) $\mathrm{k}=7.5$ GSDG

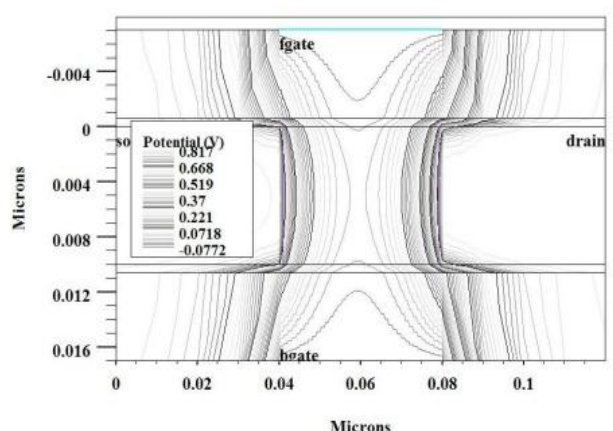

(d) $\mathrm{k}=50$

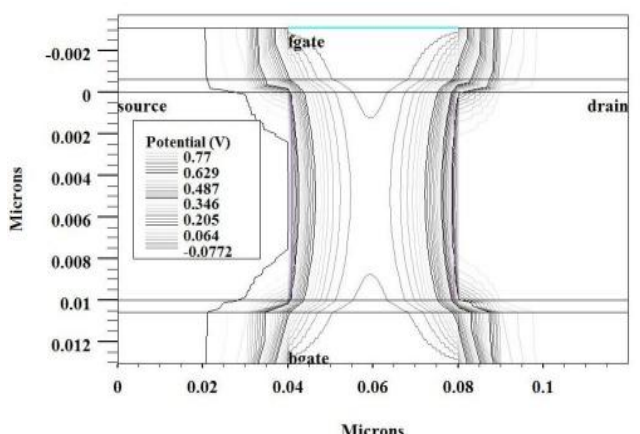

(f) $\mathrm{k}=24$ GSDG

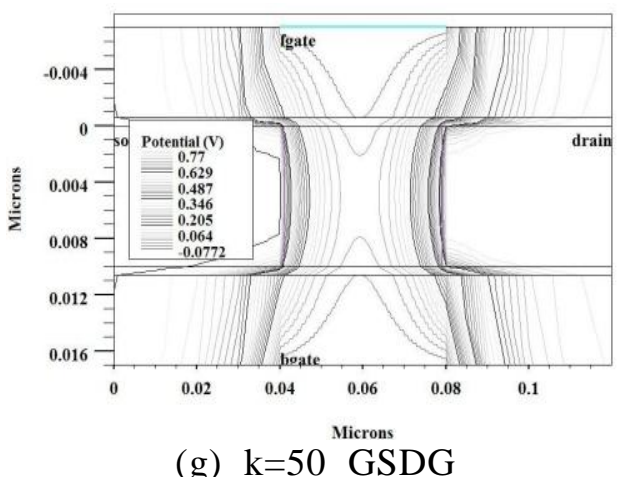

(g) $\mathrm{k}=50$ GSDG

Figure 6. The simulated results of the potential distribution in the gate insulator for different configurations keeping EOT as $1.1 \mathrm{~nm}$ in each case.

Figure for single layer configuration with $k=3.9$ (a), for single layer configuration with $k=7.5$ (b), for single layer configuration with $k=24$ (c), for single layer configuration with $k=50$ (d), for double layer/GS with $k=7.5$ (e), for double layer/GS with $k=24$ (f) and for double layer/GS with $k=50$ (g)

Figure 6 shows the potential distribution of different device structures by considering various gate dielectric materials to investigate the effect of the fringing fields from source and drain through the gate insulator. By comparing the single layer structures shown in Figure 6 (b), (c) and (d), it is observed that with increasing k value (i.e. for $\mathrm{k}=7.5,24$ and 50), the electric fields from the source to the drain spread into the channel area. This results in lowering of the channel barrier potential and induces the short channel effects. However, from the structures shown in Figure 6 (e), (f) and (g) 
with double layer/GS, the electric field lines gradually decreases from the channel. As a result the electrostatic interiority of the device is maintained.

\section{Conclusion}

The impact of high-k gate dielectrics on short channel effects in both single and double layer gate oxide structures have been studied. The degradation in short channel performance of the device is analyzed by using ATLAS 2D device simulator. This paper investigates the effect of fringing fields from the source and drain to the channel region for different structures with varying dielectric permittivity. It is found that the degradation in short channel performance using high-k dielectrics is mainly due to the fringing fields from the gate to the source/drain regions. This work also shows that better short channel performance can be achievable by taking GS/double layer configurations for the DGMOSFET. It is possible to suppress the fringing fields, $\mathrm{I}_{\text {off }}$, SS and DIBLvalues \& also to improve the performances of the nano scale DGMOSFET by considering a gate stack architecture using a thick layer of high-k dielectric material above a thin layer of $\mathrm{SiO}_{2}$ maintaining constant EOT.

\section{References}

[1] T. Skotnicki and A. James, "Hutchby, TsuJae King", H. S. Philip Wong, Frederic Boeuf, IEEE Circuits \& Devices Magazine, no. 16, (2005).

[2] F. Djeffal, M. Meguellati and A. Benhaya, "Physica E", vol. 41, no. 1872, (2009).

[3] K. F. Albertin, M. A. Valle and I. Pereyra, "Journal Integrated Circuits and Systems", vol. 2, no. 89, (2007).

[4] G. Ribes, J. Mitard, M. Denais, S. Bruyere, F. Monsieur, C. Parthasarathy, E. Vincent and G. Ghibaudo, IEEE Transactions On Device and Materials Reliability", vol. 5, no. 5, (2005).

[5] S. A. Campbell, D. C. Gilmer, X. Wang, M. Hsieh, H. -S. Kim, W. L. Gladfelter and J. Yan, "IEEE Transactions on Electron Devices", vol. 44, no. 104, (1997).

[6] B. Cheng, M. Cao, R. 1Rao, AnandInani, P. V. Voorde, W. M. Greene, J. M. C. Stork, Z. Yu, P. M. Zeitzoff and J. C. S. Woo, "IEEE Transactions on Electron Devices", vol. 46, no. 1537, (1999).

[7] A. Ohata, "Solid State Electronics", vol. 48, no. 345, (2004).

[8] B. H. Lee, S. C. Song, R. Choi and P. Kirsch, "IEEE Transactions on Electron Devices, vol. 55, no. 8, (2008).

[9] R. K. Sharma, M. Gupta and R. S. Gupta, "IEEE Transactions on Electron Devices", vol. 58, no. 2936, (2011).

[10] F. Zaunert, R. Endres, Y. Stefanov and U. Schwalke, "Journal of Telecommunications and Information Technology", vol. 2, no. 78, (2007).

[11] K. Kakushima, K.Tachi, M. Adachi, K. Okamoto, S. Sato, J. Song, T. Kawanago, P. Ahmet, K.Tsutsui, N. Sugii, T. Hattori and H. Iwai, "Solid-State Electronics", vol. 54, no. 715, (2010).

[12] ATLAS manual: SILVACO Int. Santa Clara, (2008). 
International Journal of Advanced Science and Technology Vol.65 (2014) 